\title{
Abnormal corticospinal excitability in patients with disorders of consciousness
}

\author{
Natallia Lapitskaya ${ }^{\mathrm{a}, \mathrm{b}, \mathrm{c}, *, 1}$, Olivia Gosseries ${ }^{\mathrm{b}, 1}$, Victor De Pasqua ${ }^{\mathrm{c}, \mathrm{d}}$, Asger Roer Pedersen ${ }^{\mathrm{a}}$, \\ Joergen Feldbaek Nielsen ${ }^{\mathrm{a}}$, Alain Maertens de Noordhout ${ }^{\mathrm{c}, \mathrm{d}}$, Steven Laureys ${ }^{\mathrm{b}, \mathrm{c}}$ \\ ${ }^{a}$ Research Department, Hammel Neurorehabilitation and Research Centre, Voldbyvej 15, 8450 Hammel, Denmark \\ ${ }^{\mathrm{b}}$ Coma Science Group, Cyclotron Research Centre, University of Liege, Sart-Tilman B30, 4000 Liege, Belgium \\ ${ }^{\mathrm{c}}$ Neurology Department, University of Liege, Sart-Tilman B30, 4000 Liege, Belgium \\ ${ }^{\mathrm{d}}$ Centre Hospitalier Regional de la Citadelle Boulevard du 12ème de Ligne, 1. 4000 Liège, Belgium
}

\section{A R T I C L E I N F O}

\section{Article history:}

Received 24 March 2012

Received in revised form

4 January 2013

Accepted 5 January 2013

Available online $\mathrm{xxx}$

\section{Keywords:}

Vegetative state

Unresponsive wakefulness syndrome

Minimally conscious state

Brain injury

Transcranial magnetic stimulation

Motor evoked potentials

Somatosensory evoked potentials

Short latency afferent inhibition

\begin{abstract}
A B S T R A C T
Background: Transcranial magnetic stimulation (TMS) has been frequently used to explore changes in the human motor cortex in different conditions, while the extent of motor cortex reorganization in patients in vegetative state (VS) (now known as unresponsive wakefulness syndrome, UWS) and minimally conscious (MCS) states due to severe brain damage remains largely unknown.

Objective/hypothesis: It was hypothesized that cortical motor excitability would be decreased and would correlate to the level of consciousness in patients with disorders of consciousness.

Methods: Corticospinal excitability was assessed in 47 patients ( 24 VS/UWS and 23 MCS) and 14 healthy controls. The test parameters included maximal peak-to-peak $M$-wave $\left(M_{\max }\right)$, F-wave persistence, peripheral and central motor conduction times, sensory (SEP) and motor evoked (MEP) potential latencies and amplitudes, resting motor threshold (RMT), stimulus/response curves, and short latency afferent inhibition (SAI). TMS measurements were correlated to the level of consciousness (assessed using the Coma Recovery Scale-Revised).

Results: On average, the patient group had lower $M_{\max }$, lower MEP and SEP amplitudes, higher RMTs, narrower stimulus/response curves, and reduced SAI compared to the healthy controls $(P<0.05)$. The SAI alterations were correlated to the level of consciousness $(P<0.05)$.

Conclusions: The findings demonstrated the impairment of the cortical inhibitory circuits in patients with disorders of consciousness. Moreover, the significant relationship was found between cortical inhibition and clinical consciousness dysfunction.
\end{abstract}

(c) 2013 Elsevier Inc. All rights reserved.

\section{Introduction}

Following severe brain damage, disorders of consciousness (DOC), such as vegetative state (VS) (now known as unresponsive wakefulness syndrome, UWS) [1] or minimally conscious state

Financial disclosure: NL was supported by the European Neurological Society Fellowship and Danish Medical Research Council. This research was funded by the Belgian National Funds for Scientific Research (FNRS), the European Commission (DISCOS, Mindbridge, DECODER \& COST), the French Speaking Community Concerted Research Action, the James McDonnell Foundation, the Mind Science Foundation and the University of Liège.

Conflict of interest: The authors report no economical conflicts of interest.

* Corresponding author. Research Department, Hammel Neurorehabilitation and Research Centre, Voldbyvej 15, 8450 Hammel, Denmark. Tel.: +45 8762 3573; fax: +4587623305 .

E-mail address: natlap@rm.dk (N. Lapitskaya).

1 Natallia Lapitskaya and Olivia Gosseries contributed equally to this work.
(MCS) have been linked to poor long term prognosis [2]. The recovery of consciousness could be related to the number of surviving neurons and the functional integrity of long-range cortico-cortical and cortico-thalamo-cortical connections [3]. Transcranial magnetic stimulation (TMS) is an objective method to assess the motor cortex excitability and the integrity of motor pathways [4]. Several studies indicate that cortical injuries, independent of their etiology, can lead to a reduced strength of inhibitory neurotransmission [5-8]. Paired TMS has been used for over 20 years to investigate recovery of motor function in stroke patients [9]. Cortical excitability is also shown to correlate with the severity of brain damage in patients with diffuse traumatic brain injury (TBI) [10-14] and to be related to the clinical recovery in mild to moderate TBI [15]. By coupling peripheral nerve stimulation with TMS, it is possible to modify the excitability of the motor cortex [16]. This effect, named short-latency afferent inhibition (SAI), is produced by inhibitory interactions within the cerebral cortex [17]. 
SAI is reduced in cholinergic forms of dementia, while it is normal in non-cholinergic forms of dementia [18]. The amount of SAI was found to be decreased in patients with cerebral autosomal dominant arteriopathy with subcortical infarcts and leukoencephalopathy and cognitive impairment [19]. Changes in cortical excitability in patients with DOC due to severe cerebral damage remain largely unknown [20]. To date, only one study has reported significant differences in motor threshold (MT) levels in persistently unresponsive post-comatose patients with brain injury due to anoxia and trauma [21]. Therefore, this investigation was designed to evaluate the cortical motor phenomena that indicate the strength and integrity of corticospinal pathways in patients with DOC due to severe brain damage. Moreover, we wanted to assess the relationship between SAI measures in the motor cortex and the clinical measures of consciousness.

\section{Hypothesis}

It was hypothesized that cortical motor excitability would be decreased and would correlate to the level of consciousness in patients with DOC.

\section{Methods and patients}

\section{Participants}

Forty seven patients with severe brain damage (trauma, $n=22$; anoxic-ischemic encephalopathy, $n=16$; ischemic stroke, $n=2$; hemorrhagic stroke, $n=2$; subarachnoidal hemorrhage, $n=4$; encephalitis, $n=1$ ) were prospectively included and met the criteria $[22,23$ ] defining VS/UWS $(n=23 ; 16$ males; mean age \pm SD $45 \pm 22$ years; mean time since injury \pm SD $16 \pm 53$ months), or MCS ( $n=24 ; 19$ males; mean age \pm SD $41 \pm 17$ years; mean time since injury \pm SD $36 \pm 65$ months). MRI was performed within a week of TMS assessments. See Supplemental Table for individual MRI descriptions and patient clinical characteristics. All patients were screened in order to ensure there were no contraindications to TMS [24]. No sedative medication was administered prior to the TMS measurements. Thirteen patients received no centrally acting drugs, 21 patients received one centrally acting drug (antidepressant $(n=1)$, anticonvulsant $(n=8)$, benzodiazepine $(n=2)$, antispasticity drug $(n=10)$, and 13 patients received a combination of two or more of these drugs. The administration of these drugs could not be stopped for clinical reasons. Fourteen healthy volunteers ( 8 males; mean age \pm SD $33 \pm 11$ years) were also tested.

The study was approved by the Ethics Committee of the Liège University and Liège University Hospital and written informed consent was obtained from the patient's legal guardians and healthy participants.

\section{Clinical assessment}

Coma Recovery Scale-Revised (CRS-R) [25] assessment was performed by an experienced neuropsychologist the day of, day before and day after the TMS sessions (the highest score was chosen for further analyses). The CRS-R consists of six subscales (auditory, visual, motor and oromotor/verbal functions, communication and arousal) and the maximum score is 23. In the current study, mean total CRS-R score \pm SD was $4 \pm 1$ points in VS/UWS patients and $13 \pm 5$ points in MCS patients. Mean motor CRS-R score \pm SD was $1 \pm 1$ points in VS/UWS patients and $3 \pm 2$ points in MCS patients $(0=$ none/flaccid; 1 = abnormal posturing; 2 = flexion withdrawal; 3 localization to noxious stimulation; $4=$ object manipulation; 5 = automatic motor response; 6 = functional object use).

\section{Basic electromyographic recordings (EMG)}

EMG was recorded using $\mathrm{Ag}-\mathrm{AgCl}$ surface electrodes over the first dorsal interosseous muscle (FDI). The electrodes were placed on the metacarpophalangeal joint (reference) and over the motor point (active). The EMG was recorded with the "Cambridge 1401" amplifier (Cambridge, U.K.), low pass filtered at $3 \mathrm{kHz}$ and high pass filtered at $10 \mathrm{~Hz}$. The data were stored on a computer for off-line analysis. The maximal peak-to-peak $M$-wave $\left(M_{\max }\right)$ of the FDI was measured following electrical stimulation of the ulnar nerve (0.2 ms square-wave constant current pulse) at the wrist with the cathode placed distally. The supramaximal electrical ulnar nerve stimulation was performed to elicit a recurrent discharge from spinal motor neurons ( $F$-wave). $F$-wave persistence was defined as the number of potentials with an amplitude of at least $20 \mu \mathrm{V}$ out of 20 recordings [26]. Peripheral motor conduction time (PMCT) was measured as the latency of the response from the spinal cord to the muscle, calculated using the formula $(F+M-1) / 2$, where $F$ was the shortest latency of 20 consecutive $F$-waves, $M$ was the $M$-wave latency, and 1 was the delay time (in $\mathrm{ms}$ ) for antidromic activation of the alpha motor neuron [27]. Central motor conduction time (CMCT) was calculated as the difference between the cortical MEP latency and PMCT.

\section{Somatosensory evoked potentials (SEPS)}

SEPs were registered from both hemispheres as described previously by Tokimura et al. [17] using the "Cambridge 1401" amplifier (Cambridge, U.K.). The EEG signal was low pass filtered at $3 \mathrm{kHz}$ and high pass filtered at $10 \mathrm{~Hz}$, and the data were stored on a computer for off-line analysis. Active skin electrodes were attached $3 \mathrm{~cm}$ posterior to C3 and C4 (10-20 international system) respectively for the right and left median nerve, and with reference electrode placed at Fz. A bipolar electrical stimulator was used. Test parameters were: stimulus duration $0.2 \mathrm{~ms}$; frequency $2 \mathrm{~Hz}$; intensity: $150 \%$ over the motor threshold for abductor pollicis brevis muscle. Five hundred responses were averaged. The latencies of the P14 and the N20 peaks were identified, and the amplitude of the N20 peak was measured peak-to-peak.

\section{TMS}

Both hemispheres were stimulated using TMS (200 stimulator and figure of eight shaped coil, Magstim Company Ltd, U.K.), and test responses in the contralateral FDI were recorded. In all experiments, the magnetic coil was held tangentially to the head with the handle pointing backwards and $45^{\circ}$ laterally from the midline. The coil was placed at the cranial site from which a maximum FDI response was obtained (i.e., the 'hotspot'). The hotspot was marked directly on the scalp to ensure constant coil position during the entire experimental session. The resting threshold intensity (RMT), expressed as a percentage of the maximum stimulator output (MSO), was defined as the intensity needed to produce a response of $50 \mu \mathrm{V}$ (peak-to-peak) or more in at least five out of ten consecutive stimuli in the relaxed muscle [28]. The RMT was assessed in both hemispheres. Stimulus/response curves [29] were measured with a stimulus intensity increasing in 10\% steps from RMT to $40 \%$ above RMT. Five trials were performed for each stimulus intensity. Short latency afferent inhibition (SAI) was measured in the relaxed muscle by paired-pulse stimulation paradigm [17]. Conditioning stimuli (CS) were $0.2 \mathrm{~ms}$ single pulses of electrical stimulation applied through bipolar electrodes to the median nerve at the wrist with the cathode positioned proximally. The intensity of the CS was slightly greater than motor threshold for abductor pollicis brevis muscle, and the magnetic test stimulus (TS) had an intensity of 
Table 1

Basic clinical and electrophysiological characteristics.

\begin{tabular}{lccc}
\hline Variables & Healthy & MCS & VS/UWS \\
\hline Age (years) & $33 \pm 10$ & $41 \pm 17$ & $45 \pm 22$ \\
Time since injury (months) & & $36 \pm 67$ & $17 \pm 54$ \\
CRS-R total score (points) & & $13 \pm 5$ & $4 \pm 1$ \\
CRS-R motor score (points) & & $3 \pm 2$ & $1 \pm 1$ \\
$M_{\text {max }}(\mathrm{mV})$ & $10 \pm 2.3$ & $4.8 \pm 2.0^{\mathrm{a}}$ & $5.1 \pm 2.9^{\mathrm{a}}$ \\
F-wave persistence (number) & $13.5 \pm 5.1$ & $16.1 \pm 5.8$ & $17.4 \pm 4.4$ \\
SEP P15peak latency (ms) & $14.9 \pm 1.2$ & $14.8 \pm 1.6$ & $16 \pm 2$ \\
SEP N20 peak latency (ms) & $19.3 \pm 1.1$ & $20.5 \pm 1.8$ & $20.4 \pm 1.6$ \\
SEP amplitude ( $\mu$ V) & $2.5 \pm 1.3$ & $0.93 \pm 0.6^{\mathrm{a}}$ & $0.9 \pm 0.7^{\mathrm{a}}$ \\
MEP latency (ms) & $22.3 \pm 1.2$ & $22.5 \pm 2.2$ & $22.8 \pm 2.4$ \\
PMCT (ms) & $16.4 \pm 1.2$ & $16.7 \pm 1.8$ & $16.6 \pm 1.6$ \\
CMCT (ms) & $5.9 \pm 1.2$ & $6.2 \pm 2.6$ & $6.7 \pm 3.3$ \\
RMT (\% MSO) & $39.3 \pm 7.6$ & $49.1 \pm 18.9$ & $50.9 \pm 13.3^{\mathrm{a}}$ \\
Unconditioned MEP paired- & $0.74 \pm 0.67$ & $0.88 \pm 0.75$ & $0.44 \pm 0.59^{\mathrm{a}, \mathrm{b}}$ \\
$\quad$ pulse paradigms (mV) & & & \\
\hline
\end{tabular}

Bilateral SEPs and MEPs responses were elicited in 16 MCS and 14 VS/UWS patients. Unilateral SEPs and MEPs responses were elicited in 7 MCS and 5 VS/UWS patients. In one MCS and two VS/UWS patients no SEPs or MEPs could be elicited from both hemispheres. In two VS/UWS patients SEPs were present unilaterally and MEPs responses were absent, while the recording from the other hemisphere was not performed due to clinical reasons. The presented parameters obtained from the best hemisphere in patients and from the dominant left hemisphere in healthy controls. Values are mean \pm standard deviation.

Abbreviations: MCS = minimally conscious state; VS/UWS = vegetative state/ unresponsive wakefulness syndrome; $M_{\max }=$ maximum peak-to-peak $M$-wave; $F$-wave persistence $=$ number of potentials with an amplitude of at least $20 \mu \mathrm{V}$ out of 20 F-wave recordings; PMCT = peripheral motor conduction time; $\mathrm{CMCT}=$ central motor conduction time; SEPs = sensory evoked potentials; MEPs = motor evoked potentials; RMT $=$ resting motor threshold; MSO = maximal stimulator output; uMEP $=$ unconditioned MEP to the single magnetic test stimulus.

a $P<0.05$ between VS/UWS patients and controls, and MCS patients and controls.

b $P<0.05$ between VS/UWS patients and MCS.

120\% RMT. Nine interstimuli intervals (ISIs) between CS and TS were determined relative to the latency of the N20 component of the SEP: $-2,0,+2,+4,+6,+8,+10,+12,+14$ ms. Each TMS block consisted of 50 randomized stimuli: five single TS and five CS + TS at each ISI; the interval between each TS was $8 \mathrm{~s}$.

\section{Statistical analysis}

Basic EMG parameters were analyzed with STATA (Stata version 9.2, Texas, U.S.A.). All electrophysiological parameters were analyzed separately; comparison between the parameters obtained from different hemispheres was performed by Wilcoxon matchedpairs signed-ranks tests; parameters of patient group were compared with those of the controls by means of Kruskal-Wallis and Mann-Whitney tests. Stimulus/response curves and SAI results were analyzed using a linear mixed model (SAS 9.1 procedure PROC MIXED; SAS Institute Inc., 2002-2003) with consciousness state (VS/UWS, MCS, and healthy controls) and stimulation condition (for the stimulus/response curves: from RMT to RMT $+40 \%$ in steps of $10 \%$; for the SAI: single TS and CS + TS with ISI of -2 , $0,+2,+4,+6,+8,+10,+12,+14$ ms relatively to $\mathrm{N} 20$ ) as systematic factors and subject as a random factor. The random subject factor also modeled the correlation between repeated measurements on subjects across stimuli. MEP values were log-transformed, median MEP values were summarized from five MEP trials by each stimulation condition per individual. The statistical null hypothesis of no difference between relative amplitude levels (stimulus/response curves: relative to RMT response; SAI: relative to the single TS response) was tested, and if a difference was detected, the statistical significance of each relative amplitude level was also tested. For the stimulus/response experiment, the statistical significance of the RMT was tested by adding the natural logarithm of RMT values as a continuous explanatory variable to the statistical model. Finally,

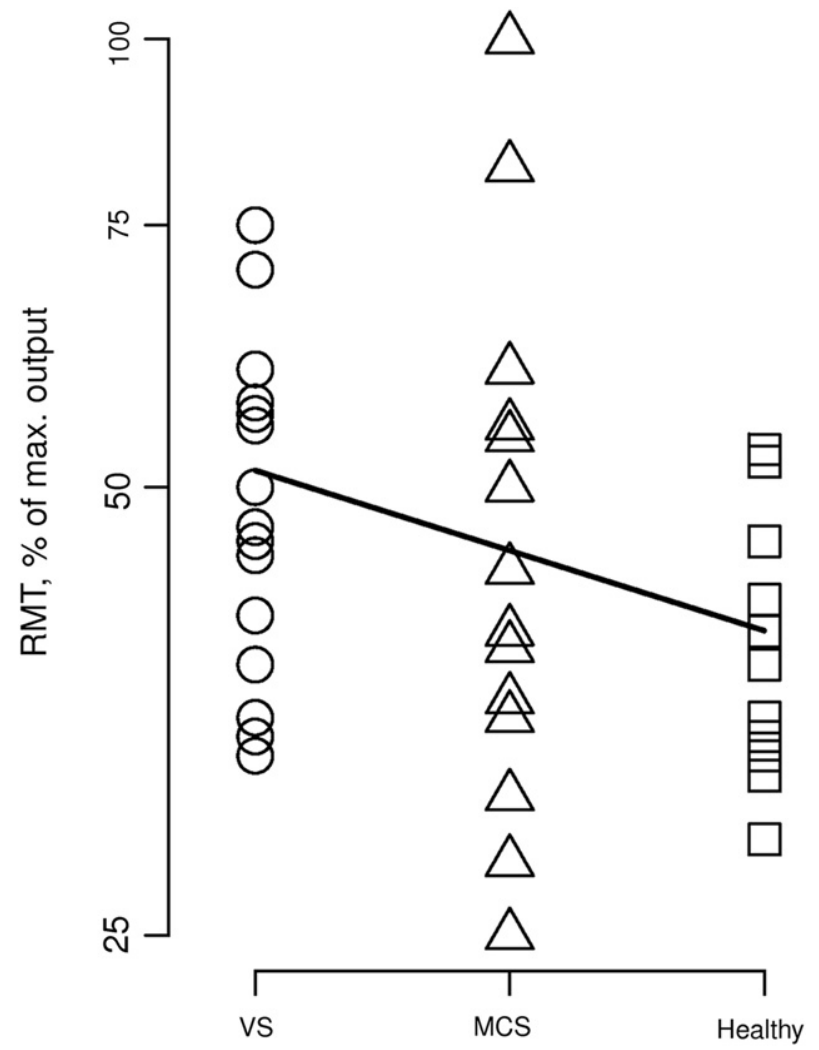

Figure 1. RMT in patients and healthy controls. RMT values were larger in VS/UWS (circles) and MCS (triangles) as compared to healthy subjects (squares), $P<0.001$. The decrease of MEP amplitude was observed with increasing RMT. The fed line represents mean RMT values. Abbreviations: RMT = resting motor threshold; VS/ UWS $=$ vegetative state/unresponsive wakefulness syndrome; MCS $=$ minimally conscious state; MEP = motor evoked potential.

for patients, the association between individual ratios of median SAI values at $\mathrm{N} 20+2 \mathrm{~ms}$ relative to the median of single TS responses and the corresponding CRS-R values was tested by the Spearman correlation coefficient. Results were considered significant at $P<0.05$.

\section{Results}

\section{Basic electrophysiological parameters}

TMS was tolerated well and no side effects were reported. Both healthy subjects and patients were instructed to relax during the entire TMS session. Neither healthy subjects nor patients were given audio-visual feedback to assist in maintaining complete relaxation. EMG traces for each trial were visually inspected offline, and trials contaminated with voluntary muscle activity were rejected from the analyses (in the recruitment curves experiment: 31 traces out of 1025 (3\%) in patients and 3 out of $350(0.9 \%)$ in healthy subjects; in the SAI experiment: 79 out of $1900(4 \%)$ in patients and 2 out of $700(0.3 \%)$ in healthy.

Bilateral SEPs and MEPs responses were elicited in $16 \mathrm{MCS}$ and $14 \mathrm{VS} / \mathrm{UWS}$ patients. Unilateral SEPs and MEPs responses were elicited in 7 MCS and 5 VS/UWS patients. In one MCS and two VS/ UWS patients no SEPs or MEPs could be elicited from both hemispheres. In two VS/UWS patients SEPs were present unilaterally and MEPs responses were absent, while the recording from the other hemisphere was not performed due to clinical reasons. 
A

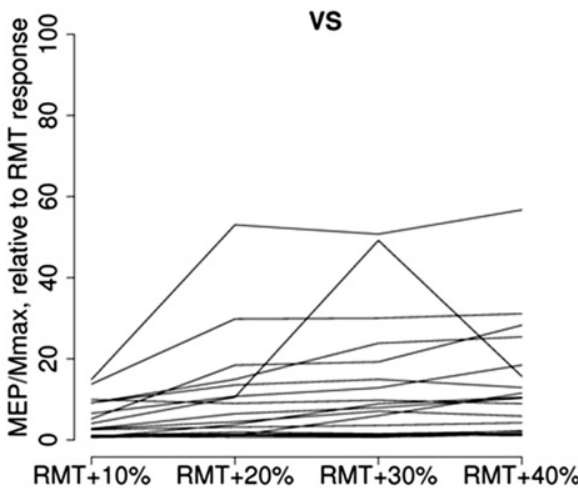

B

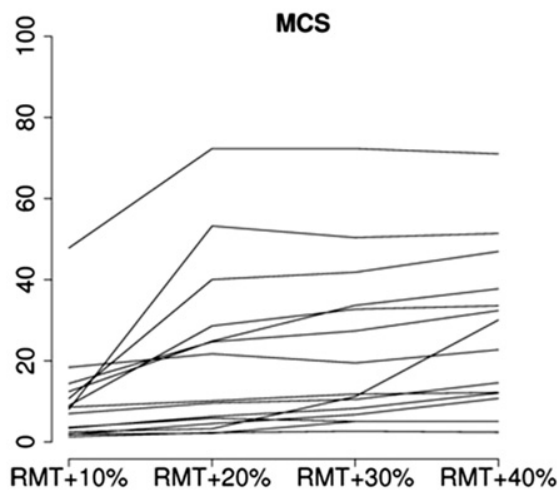

C

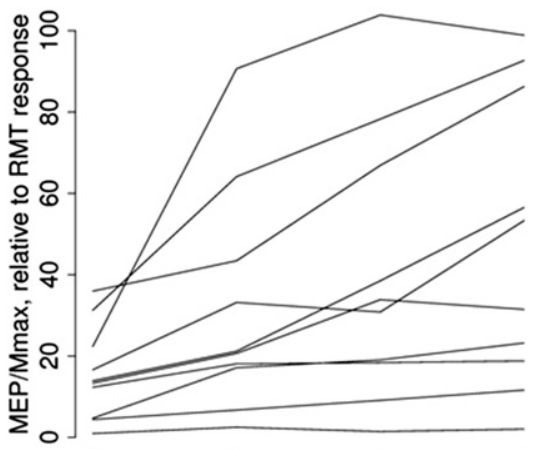

$\begin{array}{llll}\mathrm{RMT}+10 \% & \mathrm{RMT}+20 \% & \mathrm{RMT}+30 \% & \mathrm{RMT}+40 \% \mathrm{R}\end{array}$
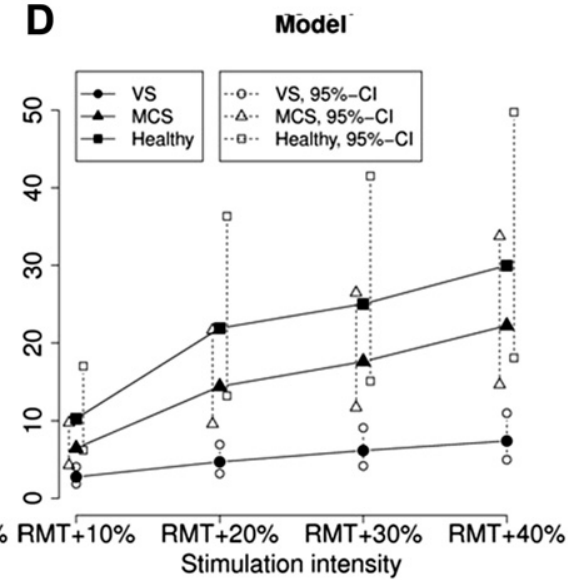

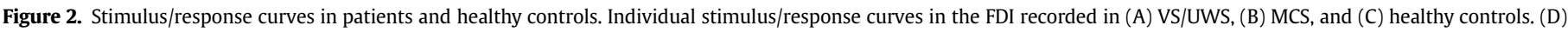

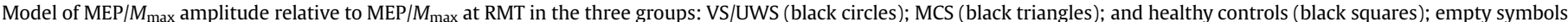

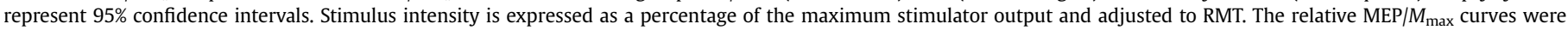

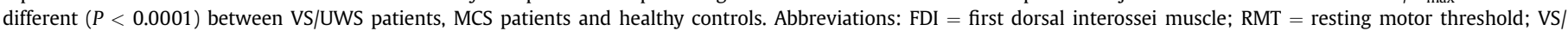

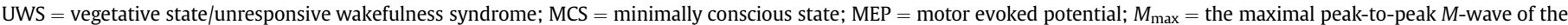
first dorsal interosseous muscle.

Table 1 summarizes basic clinical and electrophysiological parameters obtained from the best hemisphere in patients and from the dominant left hemisphere in healthy controls.

The $M_{\max }$ differed between both VS/UWS and MCS compared to healthy subjects $(P<0.001)$, but not between VS/UWS compared to MCS $(P=0.8)$. The amplitude of the N20 component of SEPs was decreased in MCS patients $(0.93 \pm 0.6 \mu \mathrm{V}, P=0.03)$, and VS/UWS patients $(0.9 \pm 0.7 \mu \mathrm{V}, P=0.04)$ compared to healthy subjects $(2.5 \pm 1.3 \mu \mathrm{V})$. Unconditioned MEP amplitude was different between both patient groups compared to healthy subjects $(P<0.05)$, and between VS/UWS compared to MCS $(P=0.02)$. The RMT differed between VS/UWS compared to healthy subjects $(P=0.01$, Fig. 1$)$, but not between MCS compared to healthy subjects $(P=0.08)$ or between VS/UWS compared to MCS $(P=0.5)$. The decrease in MEP amplitude was observed with increasing RMT (e.g., a doubling of RMT reduced MEP amplitude with 70\%). MEP amplitude tended to be higher in patients using centrally acting drugs (mean $70 \%$ (CI [-9-216\%]), but the effect was not significant $(P=0.09))$.

The MEP and SEP latencies, $F$-wave persistence, PCMT, and CMCT did not differ between patient groups and healthy controls $(P>0.05)$.

In patients with bilateral SEPs and MEPs responses, basic electrophysiological measures $\left(M_{\max }, F\right.$-wave persistence, PMCT, CMCT, SEPs latencies and amplitudes, and MEP latencies) were not different between the two recorded sides, while RMT was significantly higher in VS/UWS group ( $57.4 \pm 17.7 \%$ MSO, range 38-93) comparing to MCS group ( $49.4 \pm 18.8 \%$ MSO, range $28-100)$ and healthy controls (34.1 $\pm 10.4 \% \mathrm{MSO}$, range $23-59)$, $(P<0.05)$. The high RMT values introduced many missing values in recruitment curves and SAI paradigm and were not analyzed further. Patients with missing SEPs and/or MEPs were excluded from further analyses. The correlation between TMS measures and clinical findings in patients was analyzed for the measures obtained from the best hemisphere.

\section{Stimulus/response curves}

Stimulus/response curves exhibited a gradual increase in MEP amplitude with increasing stimulus intensities. Comparisons revealed differences between the relative MEP curves for VS/UWS, MCS and healthy controls $(P<0.0001$, Fig. 2$)$. Significant differences were shown between VS/UWS and healthy controls $(P<0.005)$, and between VS/UWS and MCS $(P<0.005)$ for $110 \%, 120 \%, 130 \%$ and $140 \%$ of RMT intensities, while no significant differences were found between MCS and healthy controls $(P>0.05)$. The model predicted that subject MEP amplitude levels were associated with RMT values $(r=-0.20, P=0.002)$ indicating a potential effect of RMT on MEP values. High values of RMT were more likely to introduce missing MEP values by exceeding the maximal output 


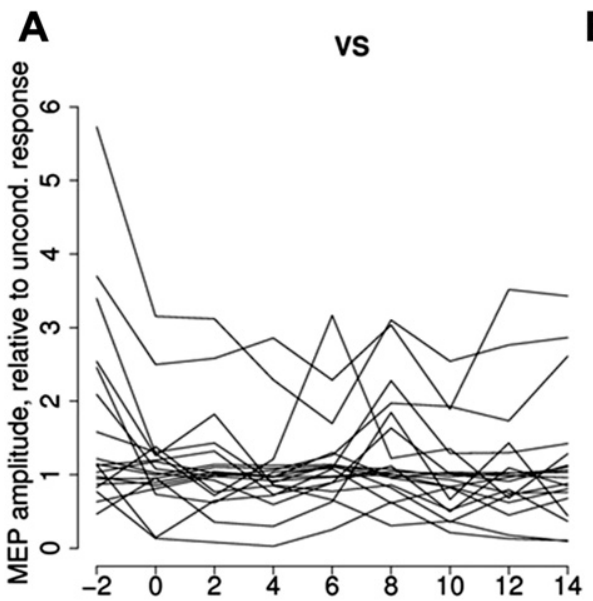

B $\quad$ MCs
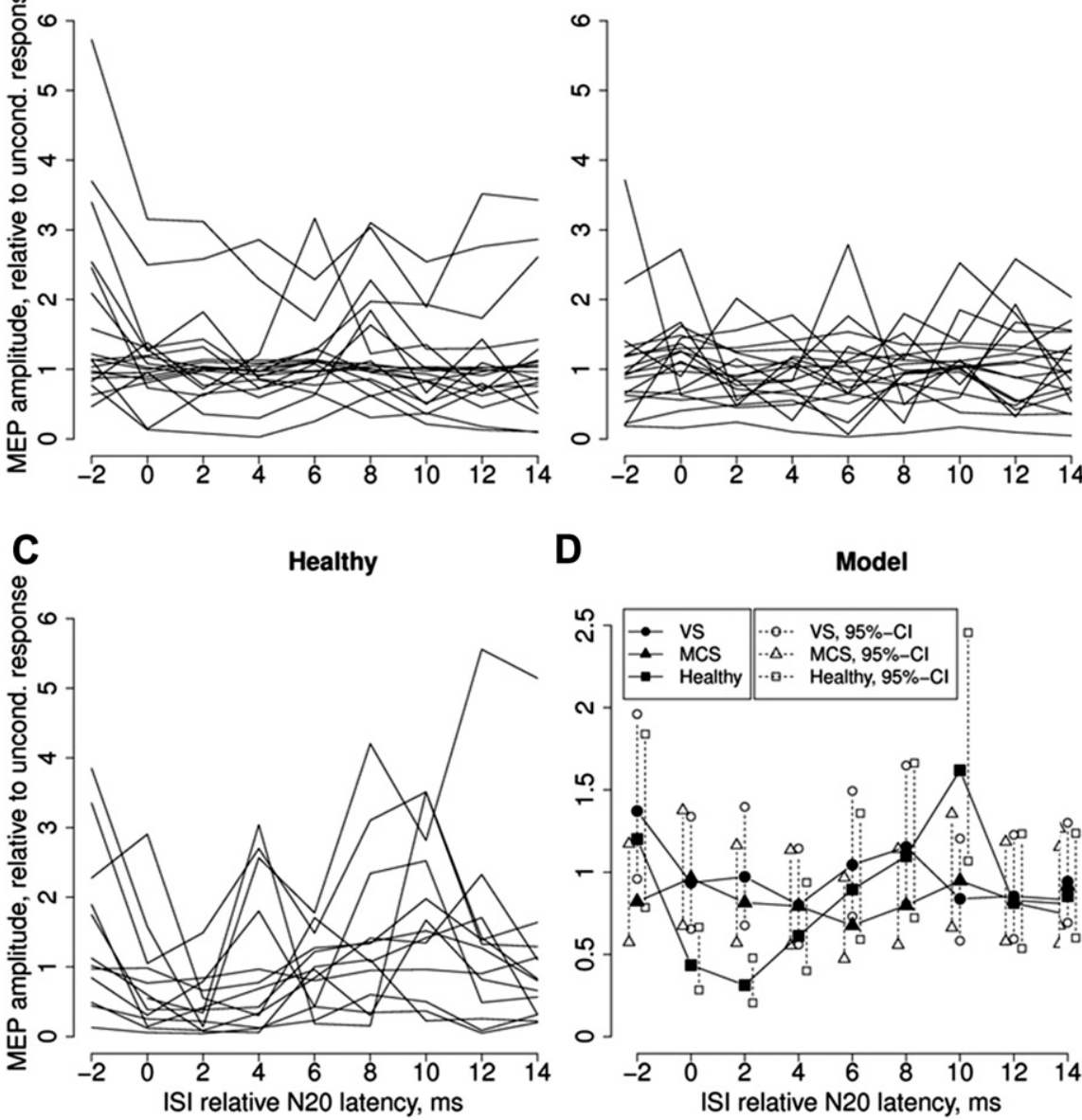

D Model

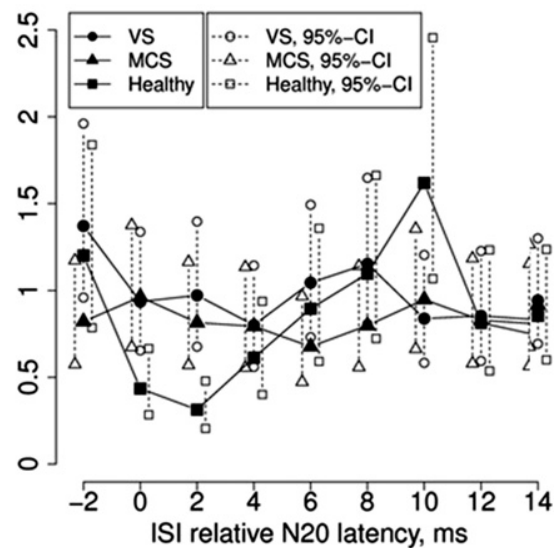

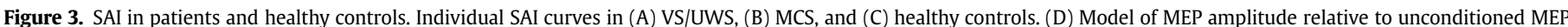

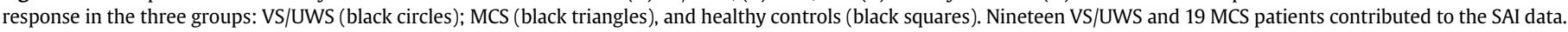

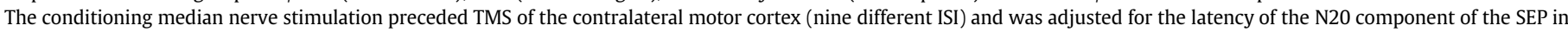

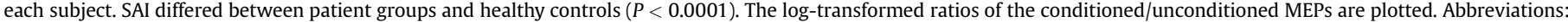

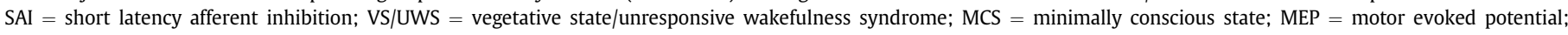
$\mathrm{SEP}=$ sensory evoked potential; ISI = interstimuli interval.

limit (i.e., introducing a negative bias on MEP values for high RMT values). This bias was presumably larger for patients.

\section{Short afferent inhibition}

Relative SAI time courses were different between groups $(P<0.0001$, Fig. 3). The responses were inhibited in healthy controls with the most pronounced inhibition when the median nerve stimulus was given at $\mathrm{N} 20+2 \mathrm{~ms}$ before the TMS stimulus $(P=0.0000)$. At this ISI interval ratios of median SAI values to the median of single TS responses were 0.31 ( $\mathrm{CI}[0.2-0.48])$ in healthy controls, 0.81 (CI [0.57-1.16]) in MCS, and 0.97 (CI [0.68-1.4]) in VS/ UWS, with a significant difference between MCS and healthy controls $(P=0.0008, \mathrm{CI}[1.5-4.54])$, and between VS/UWS and healthy controls $(P=0.00008, \mathrm{CI}[1.78-5.44])$, while the difference between VS/UWS and MCS did not reach the significance $(P=0.5$, CI $[0.72-1.99])$. There was no effect of centrally acting medication $(P=0.7)$ (inter-individual variation remained $77 \%)$. Subgroups analyses were performed according to the type of brain injury: 1) anoxia; 2) trauma; 3) non-trauma non-anoxia (including ischemic and haemorrhagic stroke, subarachnoidal hemorrhage, and encephalitis). Relative SAI time courses were different between groups ( $P=0.0395$, inter-individual variation $83 \%$, see Fig. 4 ). As shown in Fig. 5, the amount of inhibition at N20 +2 ms correlated with CRS-R total scores $(r=-0.39, P=0.02)$.

\section{Discussion}

The current study evaluated several excitatory and inhibitory phenomena in VS/UWS and MCS patients due to severe brain injury and showed that RMT, stimulus/response curves, and SAI were significantly different between patients with DOC and healthy controls. In addition, the findings revealed a relationship between SAI and clinically assessed levels of consciousness in patients with severe brain injury.

In the present study no MEPs could be elicited from both hemispheres in one MCS and four VS/UWS patients. In seven MCS and five VS/UWS patients MEPs were only elicited from one hemisphere. Both VS/UWS and MCS patients presented normal MEP and SEP latencies, $F$-wave persistence, PCMT, and CMCT. These results are in line with Moosavi et al. [21] showing that the absence of voluntary movements does not invariably predicate an abnormal motor conduction in post-comatose patients with severe brain damage. Also studies in stroke patients show that presence or 


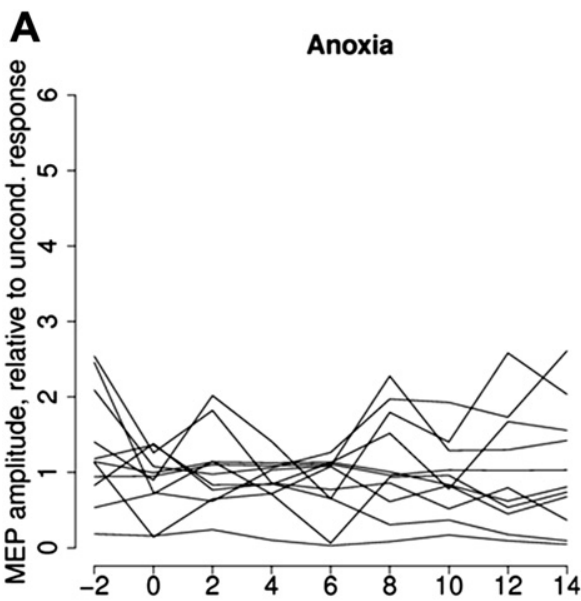

B

Trauma
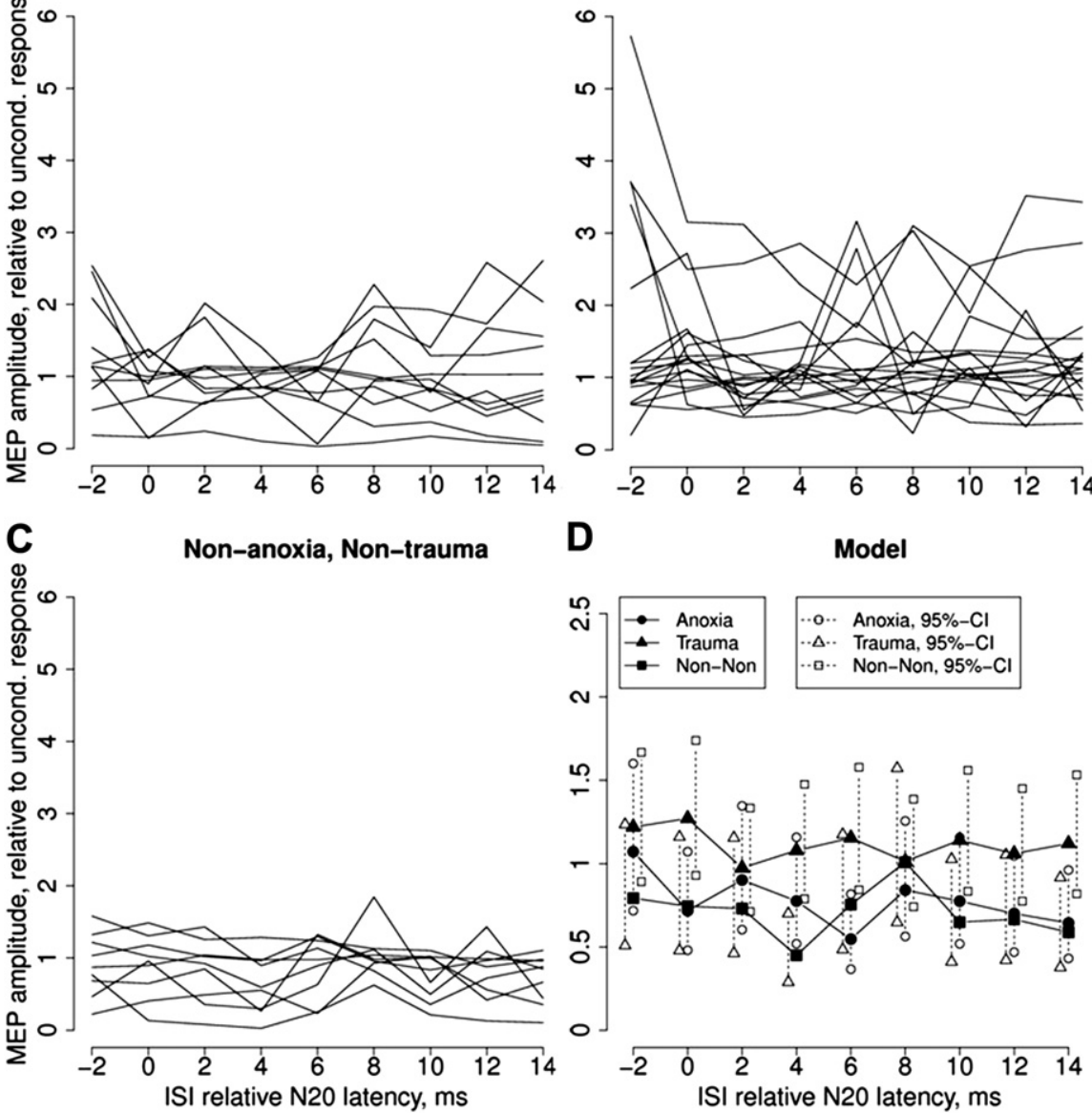

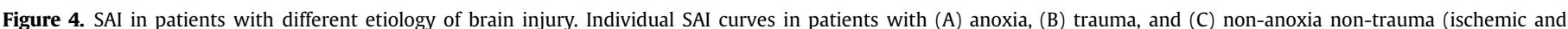

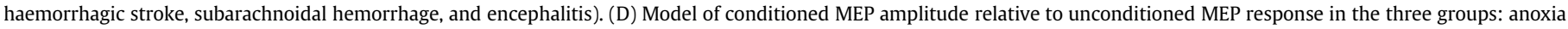

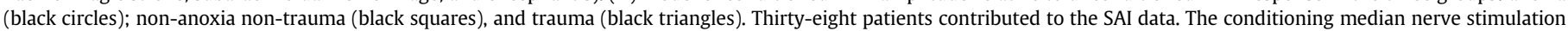

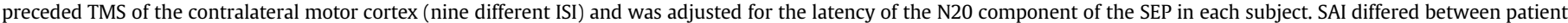

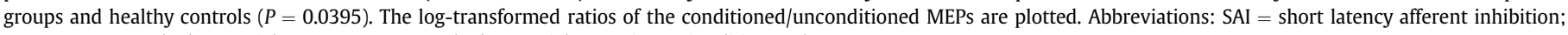
$\mathrm{MEP}=$ motor evoked potential; SEP $=$ sensory evoked potential; ISI = interstimuli interval.

absence of MEPs in the weeks and months after insult does not always anticipate clinical improvement of motor function [30,31]. The previous observations made with conventional MEPs showed that motor deficit can exist in the presence of normal corticomotoneuronal conduction times, showing that intactness of these connections is not a sufficient condition for preservation of voluntary motor activities [32]. This might indicate the selective involvement of the small pyramidal tract neurons with a relative sparing of the large fast conducting corticospinal fibers that are responsible for MEP [33]. However, both the N20 amplitude, unconditioned MEP amplitude and the $M$-wave were decreased significantly in VS/UWS patients. In agreement with previous reports in critically ill patients [34], presence of a symmetric sensory and motor axonal neuropathy is common in DOC patients in the subacute period following brain injury, while myopathic involvement is rarer and most probably is associated with neuropathies [35]. For the detection of the failure of corticospinal tract conduction, the triple stimulation technique, which consists of one transcranial magnetic and two peripheral electric stimuli (plexus and nerve) delivered along the motor pathways, might have higher sensitivity than conventional MEP or CMCT testing [36]. We observed higher RMTs in VS/UWS patients compared to healthy controls, while the difference between MCS patients and healthy subjects was not significant. These results confirm previous findings of higher MT in patients recovering from anoxia [21] and diffuse TBI $[14,15]$. The RMT is thought to reflect membrane excitability in interneuronal circuits within the motor cortex [37]. Diffusion-weighted imaging has also shown that the RMT is strongly correlated with the structural integrity of the white matter in the primary motor and premotor cortex [38]. RMT is also determined by the excitability of spinal motoneurons as discharge of these are required for an MEP to be evoked in the muscle [39]. However, we found the $F$-wave persistence to be within normal limits in both patient groups, suggesting that most of the increase in RMT was caused by reduced cortical excitability. In concurrence with these findings, a difference between VS/UWS patients and healthy controls, and between VS/UWS and MCS patients was observed in the stimulus/response curves, while no significant differences were found between MCS patients and healthy controls. Compared to RMT, stimulus/response curves assess neurons that are intrinsically less excitable and further from the centre of activation of the TMS coil [40], and are believed to reflect the size of the cortical representation and the distribution of excitability within the corticospinal projections [39].

The present study revealed a significantly reduced SAI in patients with DOC. This is in concurrence with studies demonstrating a reduced SAI in patients with diffuse TBI [10] and stroke [41]. Some evidence suggests that SAI is a cortical phenomenon [17] 


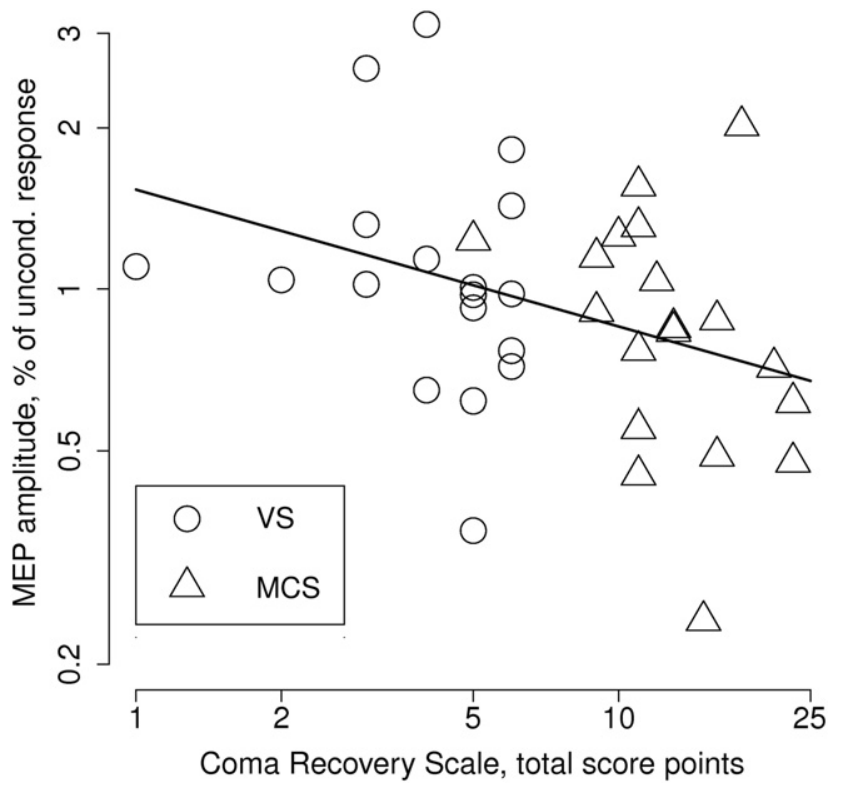

Figure 5. Correlation of SAI with clinically assessed level of consciousness. The logtransformed individual ratios of the conditioned/unconditioned MEP amplitudes at ISI $+2 \mathrm{~ms}$ (relative to N20 component of SEP) were correlated with CRS-R total scores in VS/UWS and MCS patients $(r=-0.39, P=0.02)$. Abbreviations: $\mathrm{SAI}=$ short latency afferent inhibition; MEP = motor evoked potential; SEP = sensory evoked potential; ISI = interstimuli interval; CRS-R = Coma Recovery Scale Revised [25]; VS/ UWS $=$ vegetative state/unresponsive wakefulness syndrome; MCS = minimally conscious state.

and might be related to central cholinergic activity [42,43]. The mechanisms behind SAI abnormalities in DOC remain unclear, but could possibly involve the withdrawal of tonic facilitation from the thalamus [44]. Alternatively, subcortical lesions interrupting ascending cholinergic axons may lead to cortical cholinergic denervation [45]. However, it cannot be excluded that SAI occurs via cholinergic modulation of inhibitory circuits rather than direct inhibitory cholinergic effects [41-43]. The other motor cortex excitability phenomena (e.g., short intracortical inhibition, ICI; intracortical facilitation, ICF) are also most likely impaired in patients with DOC. Reduced ICI and SAI was found in awake patients with anoxic, traumatic, and non-traumatic diffuse brain injury [46]. Moreover, the significant correlation was found between the amount of ICI and the severity of brain injury, while no correlation was found between motor cortex excitability parameters and patient functional level [46]. Both ICI and ICF impairment was found in five VS patients [47]. In addition, reduced ICI and ICF did not change in the two patients who evolved into an MCS [47]. In this respect, investigation of short and long latency intracortical inhibition and facilitation in patients with DOC would be important. A correlation between SAI and clinically assessed levels of consciousness was observed, which is in agreement with studies showing a correlation between SAI and neuropsychological measures related to long term memory and other cognitive functions in patients with Alzheimer's disease [48] and vascular dementia with microbleeds $[49,50]$. A recent study in acute stroke patients showed a correlation between SAI and long-term recovery [41]. The hypothesis that cholinergic neuronal function is impaired in brain damaged patients is also supported by preliminary pharmacological studies using acetylcholinesterase inhibitors to improve memory during acute brain injury rehabilitation [51,52].

Some limitations should be highlighted in the current study. TMS measurements could be influenced by the centrally acting drugs administered to the patients (as standard care in the rehabilitation hospital). It has been shown that benzodiazepines can reduce SAI [53], however, the influence of other centrally acting drugs on SAI has not been thoroughly investigated. While some information has been gathered on how individual drugs influence TMS [54], the effects of different drug combinations, often used in severely brain damaged patients, remain unclear. Despite this, in the current study, cortical excitability measures did not reveal drugrelated differences. A second caveat relates to the fluctuating levels of arousal frequently encountered in DOC patients, possibly altering the recorded cortical excitability parameters. In the current study, a standardized arousal facilitation protocol as defined in the CRS-R [22] was delivered to the patients. While the continuous assessment of arousal in patients with DOC is clinically challenging, future studies could use simultaneous EEG recordings to assist in identifying sleep patterns during TMS acquisitions. Finally, in the current study here was heterogeneity in the size, location, severity, and type of focal lesions in the participants with different brain injury etiology. Future studies should correlate the amount of inhibition as measured by TMS with the structural abnormalities in the cholinergic pathways [55] and the integrity of the tissue in subcortical, thalamic and brainstem regions [56] as measured using MRI (e.g., diffusion tensor imaging, fractional anisotropy, voxel-based morphometry). Nevertheless, this study demonstrates TMS changes in patients with DOC due to severe brain injury, and supports TMS as an assessment tool to provide objective complementary information and add further information to DOC as cortico-cortical disconnection syndrome [57-59].

In conclusion, the current study is the first to provide detailed information on the integrity of the corticospinal sensorimotor pathways in patients with DOC due to severe brain damage. The higher RMTs, narrower stimulus/response curves, and reduced SAI in patients with DOC might reflect a decreased cortical excitability and a reduced cortical inhibitory activity. Moreover, reduced SAI correlated with the clinically assessed level of consciousness in patients with DOC. The hypothesis that reduced SAI is related to impaired cholinergic neuronal function in patients with DOC should be explored further in the pharmacological studies. Longitudinal studies in patients with DOC are needed to assess the relationship between functional recovery of consciousness and restoration of normal corticospinal and intracortical mechanisms. This hypothesis, if confirmed, might offer prognostic surrogate markers and suggest novel therapeutic strategies.

\section{Supplementary data}

Supplementary data related to this article can be found, in the online version, at http://dx.doi.org/10.1016/j.brs.2013.01.002.

\section{References}

[1] Laureys S, Celesia GG, Cohadon F, Lavrijsen J, Leon-Carrion J, Sannita WG, et al. Unresponsive wakefulness syndrome: a new name for the vegetative state or apallic syndrome. BMC Medicine 2010 Nov;8:68.

[2] Giacino JT. The vegetative and minimally conscious states: consensus-based criteria for establishing diagnosis and prognosis. NeuroRehabilitation 2004; 19(4):293-8.

[3] Laureys S, Faymonville ME, Luxen A, Lamy M, Franck G, Maquet P. Restoration of thalamocortical connectivity after recovery from persistent vegetative state. Lancet 2000;355(9217):1790-1.

[4] Chen R, Cros D, Curra A, Di Lazzaro V, Lefaucheur JP, Magistris MR, et al. The clinical diagnostic utility of transcranial magnetic stimulation: report of an IFCN committee. Clinical Neurophysiology 2008;119(3):504-32.

[5] Schiene K, Bruehl C, Zilles K, Qu MS, Hagemann G, Kraemer M, et al. Neuronal hyperexcitability and reduction of $\operatorname{GABA}(\mathrm{A})$-receptor expression in the surround of cerebral photothrombosis. Journal of Cerebral Blood Flow and Metabolism 1996;16(5):906-14.

[6] Domann R, Hagemann G, Kraemer M, Freund HJ, Witte OW. Electrophysiological changes in the surrounding brain tissue of photochemically induced cortical infarcts in the rat. Neuroscience Letters 1993;155(1):69-72. 
[7] Neumann-Haefelin T, Witte OW. Periinfarct and remote excitability changes after transient middle cerebral artery occlusion. Journal of Cerebral Blood Flow and Metabolism 2000;20(1):45-52.

[8] Mittmann T, Luhmann HJ, Schmidtkastner R, Eysel UT, Weigel H, Heinemann U. Lseion-induced transient suppression of inhibitory function in rat neocortex in vitro. Neuroscience 1994;60(4):891-906.

[9] Talelli P, Greenwood RJ, Rothwell JC. Arm function after stroke: neurophysiological correlates and recovery mechanisms assessed by transcranial magnetic stimulation. Clinical Neurophysiology 2006;117(8):1641-59.

[10] Fujiki M, Hikawa T, Abe T, Ishii K, Kobayashi H. Reduced short latency afferent inhibition in diffuse axonal injury patients with memory impairment. Neuroscience Letters 2006;405(3):226-30.

[11] Chistyakov AV, Soustiel JF, Hafner H, Trubnik M, Levy G, Feinsod M. Excitatory and inhibitory corticospinal responses to transcranial magnetic stimulation in patients with minor to moderate head injury. Journal of Neurology, Neurosurgery and Psychiatry 2001;70(5):580-7.

[12] Chistyakov AV, Hafner H, Soustiel JF, Trubnik M, Levy G, Feinsod M. Dissociation of somatosensory and motor evoked potentials in non-comatose patients after head injury. Clinical Neurophysiology 1999;110(6):1080-9.

[13] Takeuchi N, Ikoma K, Chuma T, Matsuo Y. Measurement of transcallosal inhibition in traumatic brain injury by transcranial magnetic stimulation. Brain Injury 2006;20(9):991-6.

[14] Bernabeu M, Demirtas-Tatlidede A, Opisso E, Lopez R, Tormos JM, PascualLeone A. Abnormal corticospinal excitability in traumatic diffuse axonal brain injury. Journal of Neurotrauma 2009;26(12):2185-93.

[15] Chistyakov AV, Soustiel JF, Hafner H, Elron M, Feinsod M. Altered excitability of the motor cortex after minor head injury revealed by transcranial magnetic stimulation. Acta Neurochirurgica 1998;140(5):467-72.

[16] Di Lazzaro V, Oliviero A, Pilato F, Saturno E, Dileone M, Mazzone P, et al. The physiological basis of transcranial motor cortex stimulation in conscious humans. Clinical Neurophysiology 2004;115(2):255-66.

[17] Tokimura H, Di Lazzaro V, Tokimura Y, Oliviero A, Profice P, Insola A, et al. Short latency inhibition of human hand motor cortex by somatosensory input from the hand. The Journal of Physiology (London) 2000;523(Pt 2):503-13.

[18] Nardone R, Golaszewski S, Ladurner G, Tezzon F, Trinka E. A review of transcranial magnetic stimulation in the in vivo functional evaluation of central cholinergic circuits in dementia. Dementia and Geriatric Cognitive Disorders 2011;32(1):18-25.

[19] Manganelli F, Ragno M, Cacchio G, Iodice V, Trojano L, Silvaggio F, et al. Motor cortex cholinergic dysfunction in CADASIL: a transcranial magnetic demonstration. Clinical Neurophysiology 2008;119(2):351-5.

[20] Lapitskaya N, Coleman MR, Nielsen JF, Gosseries O, de Noordhout AM. Disorders of consciousness: further pathophysiological insights using motor cortex transcranial magnetic stimulation. Progress in Brain Research 2009; 177:191-200.

[21] Moosavi SH, Ellaway PH, Catley M, Stokes MJ, Haque N. Corticospinal function in severe brain injury assessed using magnetic stimulation of the motor cortex in man. Journal of Neurological Sciences 1999;164(2):179-86.

[22] Giacino JT, Ashwal S, Childs N, Cranford R, Jennett B, Katz DI, et al. The minimally conscious state: definition and diagnostic criteria. Neurology 2002; 58(3):349-53.

[23] Royal College of Physicians. The vegetative state: guidance on diagnosis and management. Clinical Medicine 2003;3(3):249-54.

[24] Rossi S, Hallett M, Rossini PM, Pascual-Leone A. Safety, ethical considerations, and application guidelines for the use of transcranial magnetic stimulation in clinical practice and research. Clinical Neurophysiology 2009;120(12): 2008-39.

[25] Giacino JT, Kalmar K, Whyte J. The JFK coma recovery scale-revised: measurement characteristics and diagnostic utility. Archives of Physical Medicine and Rehabilitation 2004;85(12):2020-9.

[26] Puksa L, Stalberg E, Falck B. Reference values of F wave parameters in healthy subjects. Clinical Neurophysiology 2003;114(6):1079-90.

[27] Samii A, Luciano CA, Dambrosia JM, Hallett M. Central motor conduction time: reproducibility and discomfort of different methods. Muscle and Nerve 1998; 21(11):1445-50.

[28] Rossini PM, Berardelli A, Deuschl G, Hallett M, Maertens de Noordhout AM, Paulus W, et al. Applications of magnetic cortical stimulation. The International Federation of Clinical Neurophysiology. Electroencephalography and Clinical Neurophysiology Supplement 1999;52:171-85.

[29] Abbruzzese G, Trompetto C. Clinical and research methods for evaluating cortical excitability. Journal of Clinical Neurophysiology 2002;19(4):307-21.

[30] Delvaux V, Alagona G, Gerard P, De Pasqua V, Pennisi G, de Noordhout AM. Post-stroke reorganization of hand motor area: a 1-year prospective follow-up with focal transcranial magnetic stimulation. Clinical Neurophysiology 2003; 114(7):1217-25.

[31] Pennisi G, Rapisarda G, Bella R, Calabrese V, de Noordhout AM, Delwaide PJ. Absence of response to early transcranial magnetic stimulation in ischemic stroke patients - prognostic value for hand motor recovery. Stroke 1999; 30(12):2666-70.

[32] Homberg V, Stephan KM, Netz J. Transcranial stimulation of motor cortex in upper motor neuron syndrome: its relation to the motor deficit. Electroencephalography and Clinical Neurophysiology 1991;81(5):377-88.

[33] Di Lazzaro V, Oliviero A, Profice P, Ferrara L, Saturno E, Pilato F, et al. The diagnostic value of motor evoked potentials. Clinical Neurophysiology 1999; 110(7):1297-307.
[34] De Jonghe B, Sharshar T, Lefaucheur JP, Authier FJ, Durand-Zaleski I, Boussarsar $\mathrm{M}$, et al. Paresis acquired in the intensive care unit - a prospective multicenter study. JAMA 2002;288(22):2859-67.

[35] Bagnato S, Boccagni C, Angelo AS, Prestandrea C, Ronnano MC, Galardi G Neuromuscular involvement in vegetative and minimally conscious states following acute brain injury. Journal of the Peripheral Nervous System 2011; 16(4):315-21.

[36] Magistris MR, Rosler KM, Truffert A, Landis T, Hess CW. A clinical study of motor evoked potentials using a triple stimulation technique. Brain 1999;122: 265-79.

[37] Di Lazzaro V, Oliviero A, Pilato F, Mazzone P, Insola A, Ranieri F, et al. Corticospinal volleys evoked by transcranial stimulation of the brain in conscious humans. Neurological Research 2003;25(2):143-50.

[38] Kloppel S, Baumer T, Kroeger J, Koch MA, Buchel C, Munchau A, et al. The cortical motor threshold reflects microstructural properties of cerebral white matter. NeuroImage 2008;40(4):1782-91.

[39] Siebner HR, Rothwell J. Transcranial magnetic stimulation: new insights into representational cortical plasticity. Experimental Brain Research 2003;148(1): $1-16$.

[40] Vallssole J, Pascualleone A, Brasilneto JP, Cammarota A, McShane L, Hallett M. Abnormal facilitation of the response to transcranial magnetic stimulation in patients with Parkinsons disease. Neurology 1994;44(4):735-41.

[41] Di Lazzaro V, Profice P, Pilato F, Capone F, Ranieri F, Florio L, et al. The level of cortical afferent inhibition in acute stroke correlates with long-term functional recovery in humans. Stroke 2012;43(1):250-U493.

[42] Di Lazzaro V, Oliviero A, Tonali PA, Marra C, Daniele A, Profice P, et al Noninvasive in vivo assessment of cholinergic cortical circuits in AD using transcranial magnetic stimulation. Neurology 2002;59(3):392-7.

[43] Di Lazzaro V, Pilato F, Dileone M, Saturno E, Oliviero A, Marra C, et al. In vivo cholinergic circuit evaluation in frontotemporal and Alzheimer dementias. Neurology 2006;66(7):1111-3.

[44] Oliviero A, Leon AM, Holler I, Vila JF, Siebner HR, Della Marca G, et al. Reduced sensorimotor inhibition in the ipsilesional motor cortex in a patient with chronic stroke of the paramedian thalamus. Clinical Neurophysiology 2005 116(11):2592-8

[45] Selden NR, Gitelman DR, Salamon-Murayama N, Parrish TB, Mesulam MM Trajectories of cholinergic pathways within the cerebral hemispheres of the human brain. Brain 1998;121:2249-57.

[46] Lapitskaya N, Moerk SK, Gosseries O, Nielsen JF, de Noordhout AM. Corticospinal excitability in patients with anoxic, traumatic, and non-traumatic diffuse brain injury. Brain Stimulation 2012 Apr 18 [Epub ahead of print].

[47] Bagnatoa S, Boccagnia C, Sant'Angeloa A, Prestandreaa C, Rizzob S, Galardi G. Patients in a vegetative state following traumatic brain injury display a reduced intracortical modulation. Clinical Neurophysiology 2012;123(10): 1937-41, http://dx.doi.org/10.1016/j.clinph.2012.03.014.

[48] Di Lazzaro V, Pilato F, Dileone M, Pfofice P, Marra C, Ranieri F, et al. In vivo functional evaluation of central cholinergic circuits in vascular dementia. Clinical Neurophysiology 2008;119(11):2494-500.

[49] Nardone R, Bergmann J, Tezzon F, Ladurner G, Golaszewski S. Cholinergic dysfunction in subcortical ischaemic vascular dementia: a transcranial magnetic stimulation study. Journal of Neural Transmission 2008;115(5): 737-43.

[50] Nardone R, De Blasi P, Seidl M, Holler Y, Caleri F, Tezzon F, et al. Cognitive function and cholinergic transmission in patients with subcortical vascular dementia and microbleeds: a TMS study. Journal of Neural Transmission 2011; 118(9):1349-58.

[51] Masanic CA, Bayley MT, vanReekum R, Simard M. Open-label study of donepezil in traumatic brain injury. Archives of Physical Medicine and Rehabilitation 2001;82(7):896-901.

[52] Taverni JP, Seliger G, Lichtman SW. Donepezil mediated memory improvement in traumatic brain injury during post acute rehabilitation. Brain Injury 1998;12(1):77-80.

[53] Di Lazzaro V, Oliviero A, Saturno E, Dileone M, Pilato F, Nardone R, et al. Effects of lorazepam on short latency afferent inhibition and short latency intracortical inhibition in humans. Journal of Physiology (London) 2005;564(2): 661-8.

[54] Paulus W, Classen J, Cohen LG, Large CH, Di Lazzaro V, Nitsche M, et al. State of the art: pharmacologic effects on cortical excitability measures tested by transcranial magnetic stimulation. Brain Stimulation 2008;1(3):151-63.

[55] Babiloni C, Pievani M, Vecchio F, Geroldi C, Eusebi F, Fracassi C, et al. Whitematter lesions along the cholinergic tracts are related to cortical sources of EEG rhythms in amnesic mild cognitive impairment. Human Brain Mapping 2009;30(5):1431-43.

[56] Fernandez-Espejo D, Bekinschtein T, Monti MM, Pickard JD, Junque C, Coleman MR, et al. Diffusion weighted imaging distinguishes the vegetative state from the minimally conscious state. NeuroImage 2011;54(1): $103-12$.

[57] Laureys S, Goldman S, Phillips C, Van Bogaert P, Aerts J, Luxen A, et al Impaired effective cortical connectivity in vegetative state. Neurolmage 1999; 9(4):377-82.

[58] Laureys S. The neural correlate of (un)awareness: lessons from the vegetative state. Trends in Cognitive Sciences 2005;9(12):556-9.

[59] Boly M, Garrido MI, Gosseries O, Bruno MA, Boveroux P, Schnakers C, et al. Preserved feedforward but impaired top-down processes in the vegetative state. Science 2011;332(6031):858-62. 